\title{
Significance of ROI Coding using MAXSHIFT Scaling applied on MRI Images in Teleradiology- Telemedicine
}

\author{
Pervez Akhtar ${ }^{1}$, Muhammad Iqbal Bhatti ${ }^{2}$, Tariq Javid Ali $^{1,3}$ \& Muhammad Abdul Muqeet $^{2}$ \\ ${ }^{1}$ EPE Department, NUST, PNEC, Karachi-Campus, Karachi-75350, Pakistan. ${ }^{2}$ BME Department, SSUET, Karachi-75300, Pakistan. ${ }^{3}$ ISD Department, IT \\ Division, AKU, Karachi-74800, Pakistan. Correspondence should be addressed to pervez@pnec.edu.pk, mibhatti@ssuet.edu.pk, tariqjavid@pnec.edu.pk, and \\ mabdul@ssuet.edu.pk.
}

\begin{abstract}
Within the expanding paradigm of medical imaging in Teleradiology-Telemedicine there is increasing demand for transmitting diagnostic medical imagery. These are usually rich in radiological contents and the associated file sizes are large which must be compressed with minimal file size to minimize transmission time and robustly coded to withstand required network medium. It has been reinforced through extensive research that the diagnostically important regions of medical images, the Region of Interest (ROI), must be compressed by lossless or near lossless algorithm while on the other hand, the background region be compressed with some loss of information but still recognizable using JPEG 2000 standard. We develop a compression model and present its application on MRI images. Applying on MRI images achieved higher compression ratio $16: 1$, analogously minimum transmission time, using MAXSHIFT method proved diagnostically significant and effective both objectively and subjectively.
\end{abstract}

Keywords: Image compression, MRI, ROI, MAXSHIFT

\section{INTRODUCTION}

The objectives of teleradiology-telemedicine are to improve access and to enhance overall quality of care at an affordable cost. Improved access and cost savings could be achieved by allowing a doctor to remotely examine patients or to consult with a specialist. This reduces or eliminates the time and expense of travel necessary to bring the patient to the doctor or the doctor to the patient [1]. Quality of care is improved by providing the diagnostically important images. Rigorous research in diagnostic imaging and image compression in teleradiology-telemedicine is gaining prominence all over the world, particularly in developing countries [2]. Engineers are developing technologies and tools, enabling the medical practitioners to provide efficient treatment. From the elaborate medical information, the doctor prefers to focus on certain selected region(s) of interest. Also the doctors are more comfortable with image processing and analysis solutions that offer subjective analysis of medical images than depending on the objective engineering results alone. Technology assisted, integrated diagnostic methods are of high relevance in this context [3].

An 8-bit gray scale image with $512 \times 512$ pixels requires more than $0.2 \mathrm{MB}$ of storage. If the image can be compressed by 8:1 without any perceptual distortion, the capacity of storage increased 8 times. This is significant for teleradiology-telemedicine (TT) scenario due limitations of transmission medium. If we need $\mathrm{T}$ units of time to transmit an image, then with 16:1 compression ratio the transmission time will decrease to T/16 units of time. It has been reinforced through extensive research that the diagnostically important regions of medical images must be compressed by lossless or near lossless algorithm, while on the other hand, the background region be compressed with some loss of information but still recognizable using the JPEG2000 (JP2K) standard [4]. Applying JP2K ROI coding using MAXSHIFT scaling method on MRI images achieved high compression ratio $16: 1$ with varying quantization levels $(1 / 128,1 / 64$, and $1 / 32)$ analogously reduced transmission time; the MAXSHIFT method proved very effective both objectively and subjectively.

The paper layout follows as: In section 2, we introduce lossless coding schemes and ROI coding in JP2K standard as concepts. Section 3 describes our approach in context of MAXSHIFT scaling method and the model description. In section 4 , we give experimental results and discussion. We conclude our paper in section 5.

\section{CONCEPTS}


In the medical scenario, ROI is the area of an image, which is of clinical (diagnostic) importance to the doctor [5]. Certain image specific features like the uniformity of texture, color, intensity, etc. generally characterize as region of interest. Medical images are mostly in grayscale [6]. The gray scales of an M-bit level image (where $\mathrm{M}$ can be 8, 12 or 16 bits) can be represented in the form of bit-planes [7], See Figure 1. Identifying and extracting ROI accurately is very important before coding and compressing the image data for efficient transmission or storage. In different spatial regions and identifying the ROI in the image, it is possible to compress them with different levels of reconstruction quality. This way one could accurately preserve the features needed and transmit those for medical diagnosis or for scientific measurement, while achieving high compression overall by allowing degradation of data in the un-important regions.

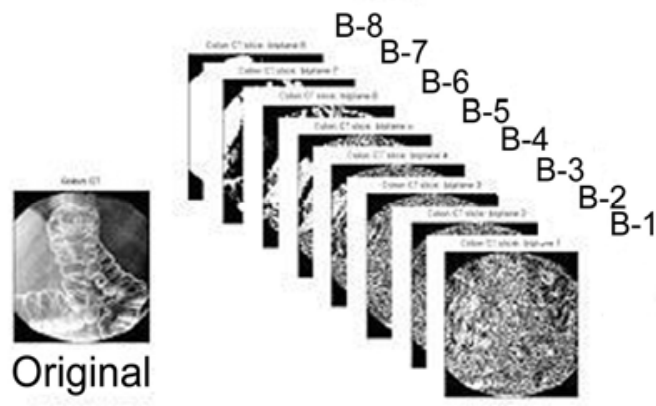

Figure.1. Colon image and its eight bitplanes.

\subsection{Lossless coding and JPEG 2000 standard}

In medical context the regionally lossless schemes have to be studied more closely. They can be any of the following based on different types of end-user/observer or context:

1) Visually lossless: non-clinical human observer.

2) Diagnostically lossless: clinical-observers; in the diagnosis significant degrees of observer dependent variations exist.

3) Quantifiably lossless: mostly non-human observer or computer assisted detection.

One important consideration here is that, what may be visually lossless or quantifiably lossless may not be diagnostically lossless [8].

Most of the commonly used methods use JP2K standard that involves the following important steps [9]. Along with these mentioned below, additional processing related to ROI mask generation and customized coding that suits the user requirement is done. Discrete Wavelet Transform (DWT) is performed on the tiles or the entire image based on size of the image [10].

1) If the ROI is identified then ROI mask is derived extracting the region indicating the set of coefficients that are required for lossless ROI reconstruction.

2) The wavelet coefficients are quantized as per desired quality of reconstruction.
3) The coefficients that are out of the ROI are scaled up/down by a specific scaling value. If there are more than one ROI, these can be multiply coded with different scaling values.

4) The resulting coefficients are progressively entropy encoded (with the most significant bit planes first). As overhead information, the scaling value assigned to the ROI and ROI mask generation but scales up the background coefficients in order to recreate the original coefficients.

\subsection{MAXSHIFT and general scaling methods}

While compressing the medical images it is important to consider ROI masking methods so as to get diagnostically important area as a lossless region. MAXSHIFT method in comparison to general scaling method supports the use of any mask since the decoder does not need to generate the mask, See Figure 2. Thus, it is possible for the encoder to include an entire subband, that is, the low-low subband, in the ROI mask and thus send a low-resolution version of the background at an early stage of the progressive transmission. This is done by scaling of all quantized transform coefficients of the entire subband. In other words, the user can decide in which subband he will start having ROI and thus, it is not necessary to wait for the whole ROI before receiving any information for the background. However, since the background coefficients are scaled down rather than scaling up ROI coefficients, this will only have the effect that in certain implementations the least significant bitplanes for the background may be lost. In other words, the background received in a degraded state (lossy) but still recognizable. The advantage is that the ROI, which is considered to be the most important part of the image, is still optimally treated while the background is allowed to have degraded quality, since it is considered to be less important.

In medical situations during compression phase, lossy schemes are not preferred. To avoid the chance of loosing any diagnostic information, a 32x32 code block size is selected with considering ROI size less than one fourth of the original image. Lossless schemes prove costly with less compression efficiencies and are ineffective in certain application domains. Regionally lossless schemes prove as a valuable/meaningful solution between the completely lossless or lossy ones. In these, lossless coding is done for the ROI and lossy coding to the less significant background image [11].

\section{OUR APPROACH}

We develop our approach based on the following observations:

1) The doctor prefers to use eyes (subjective decision) to select the region that is of importance through interactive evaluation and manual marking or selection of regions $[12,5]$. 


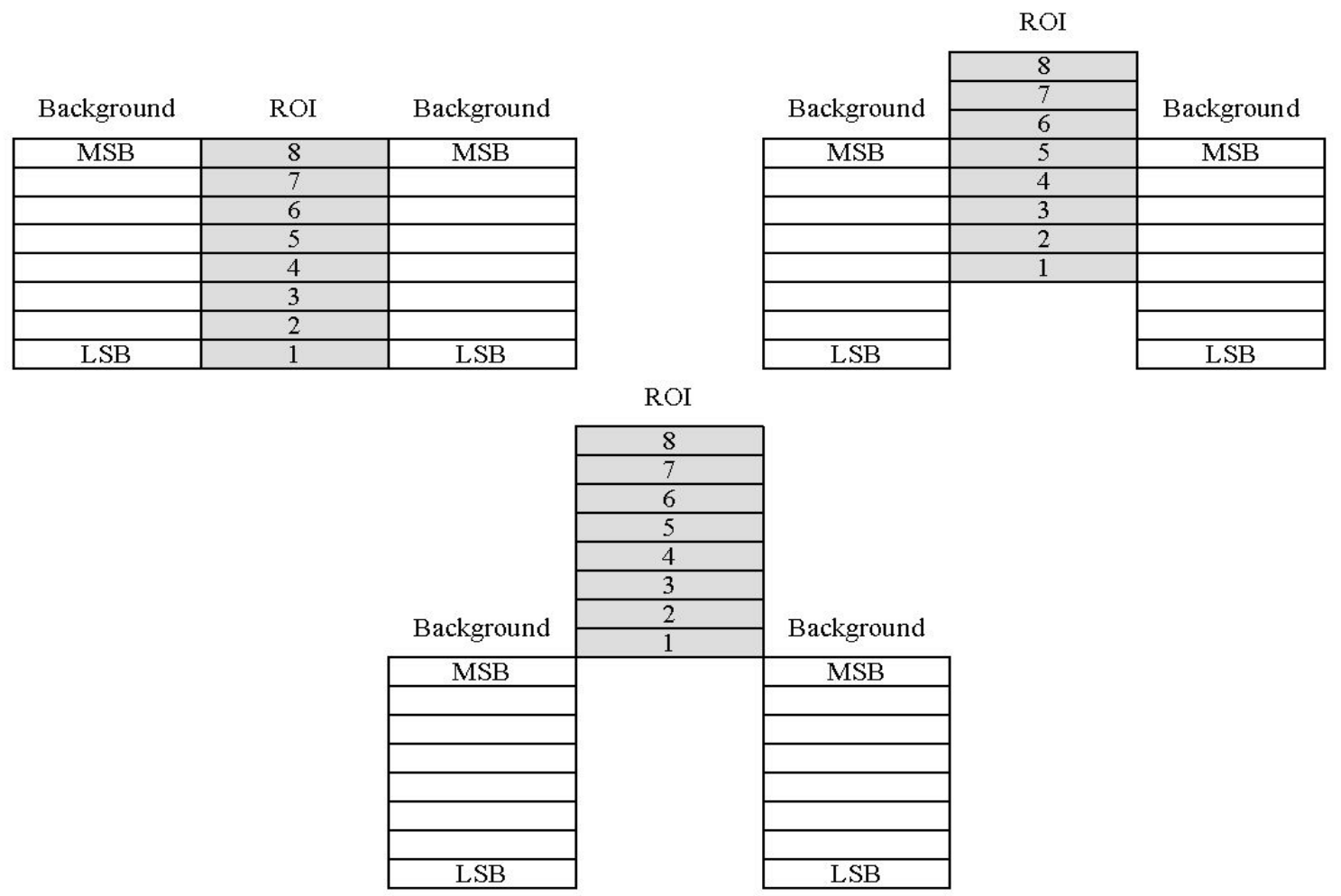

Figure 2. The MAXSHIFT method: (top left) ROI and background at the same level, (top right) general scaling method, and (bottom) MAXSHIFT method, See subsection 3.1 for details.

2) The pixels that represent the diagnostically relevant data are of interest to the doctor. These bits need not belong to visually significant data like sudden intensity variations in images.

3) The need of higher compression for a faster transmission.

An ROI is a part of an image that is coded earlier in the codestream than the rest of the image. Coding steps in the MAXSHIFT method are [13]:

1) Generate an ROI mask.

2) Find the scaling value which is greater than or equal to the largest number of magnitude bit planes for any background coefficient in any code-block in the current component.

3) Scale down all background coefficients given by mask in step (1) using the scaling value from step (2).

4) Write the scaling value into codestream.

\subsection{Transmission hierarchy}

Recent acceptance and deployment of picture archiving and communications system (PACS) [14] in hospitals and the availability of digital imaging and communications in Medicine (DICOM) medical images via PACS is and important building block of telemedicine. Figure 3 illustrates transmission hierarchy, the extension of a PACS to remote sites (imaging center); using teleradiology-telemedicine.

\section{EXPERIMENTAL RESULTS}

MRI images were obtained from a famous national health care institution. We used IrfanView [15] for image format conversion, MATLAB [16] for mathematical treatment and graphs, JJ2000 [17] for image compression, and Microsoft Office Excel to organizing the data. The performance evaluation of JP2K compression standard using MAXSHIFT method is an individual application on selected images from each of the modalities. Figures 4 and 5 show our developed compression model and an application, respectively.

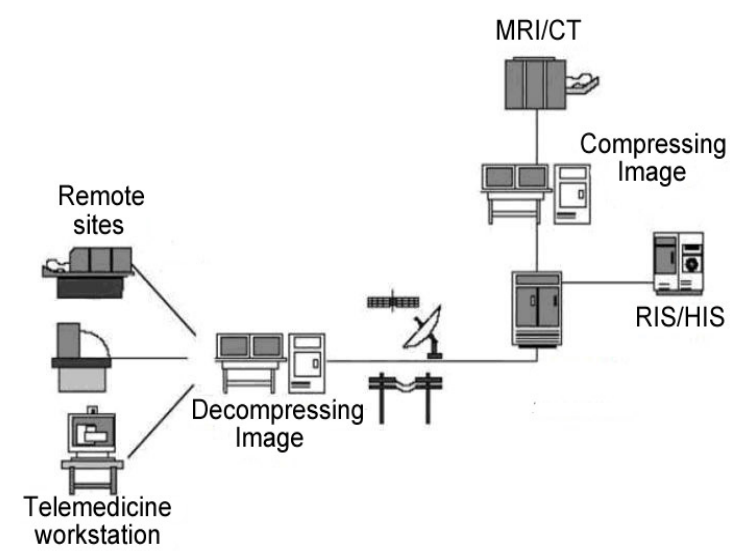

Figure 3. Transmission hierarchy. 


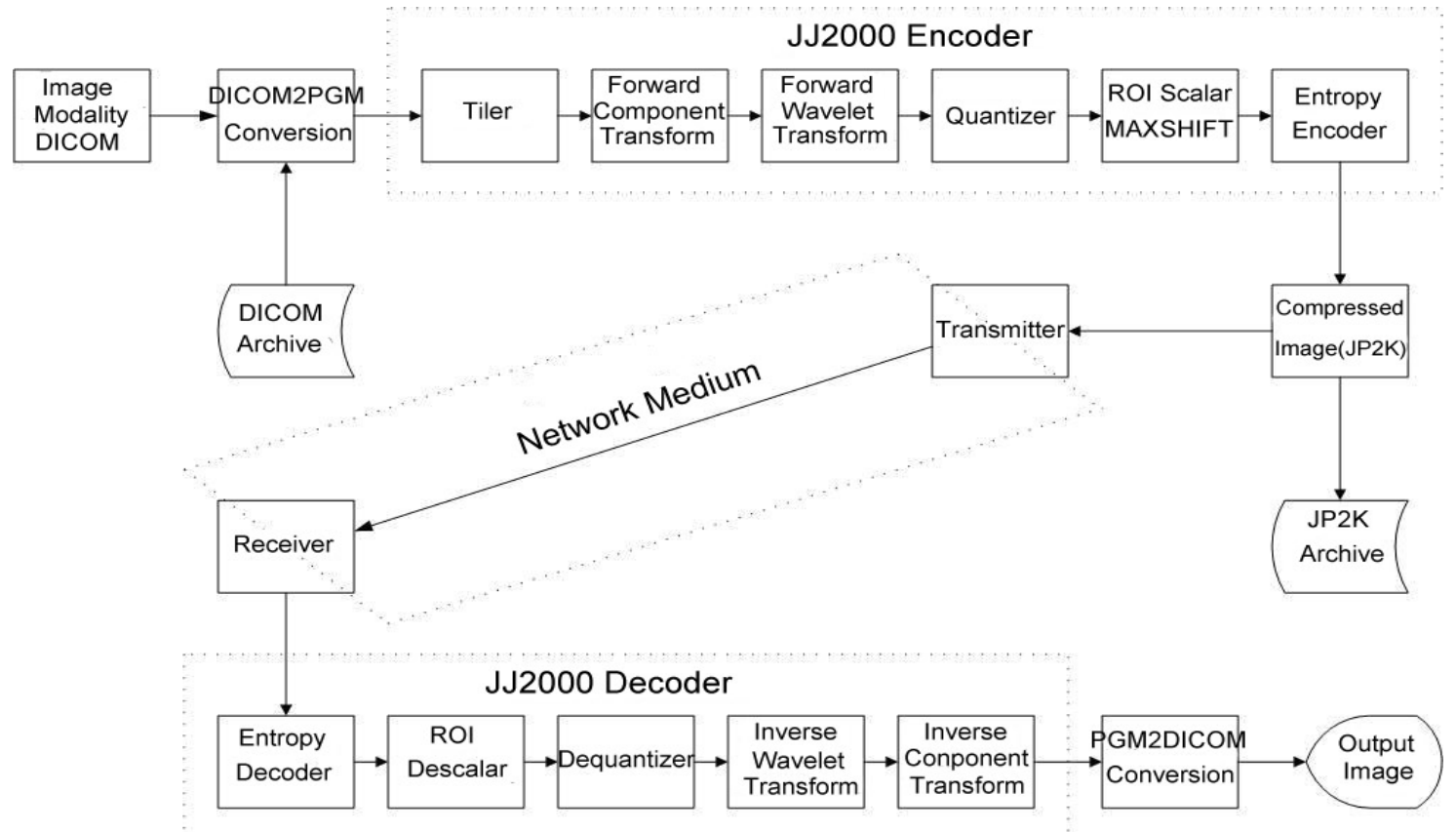

Figure 4. The compression model for TT in Fig. 3.

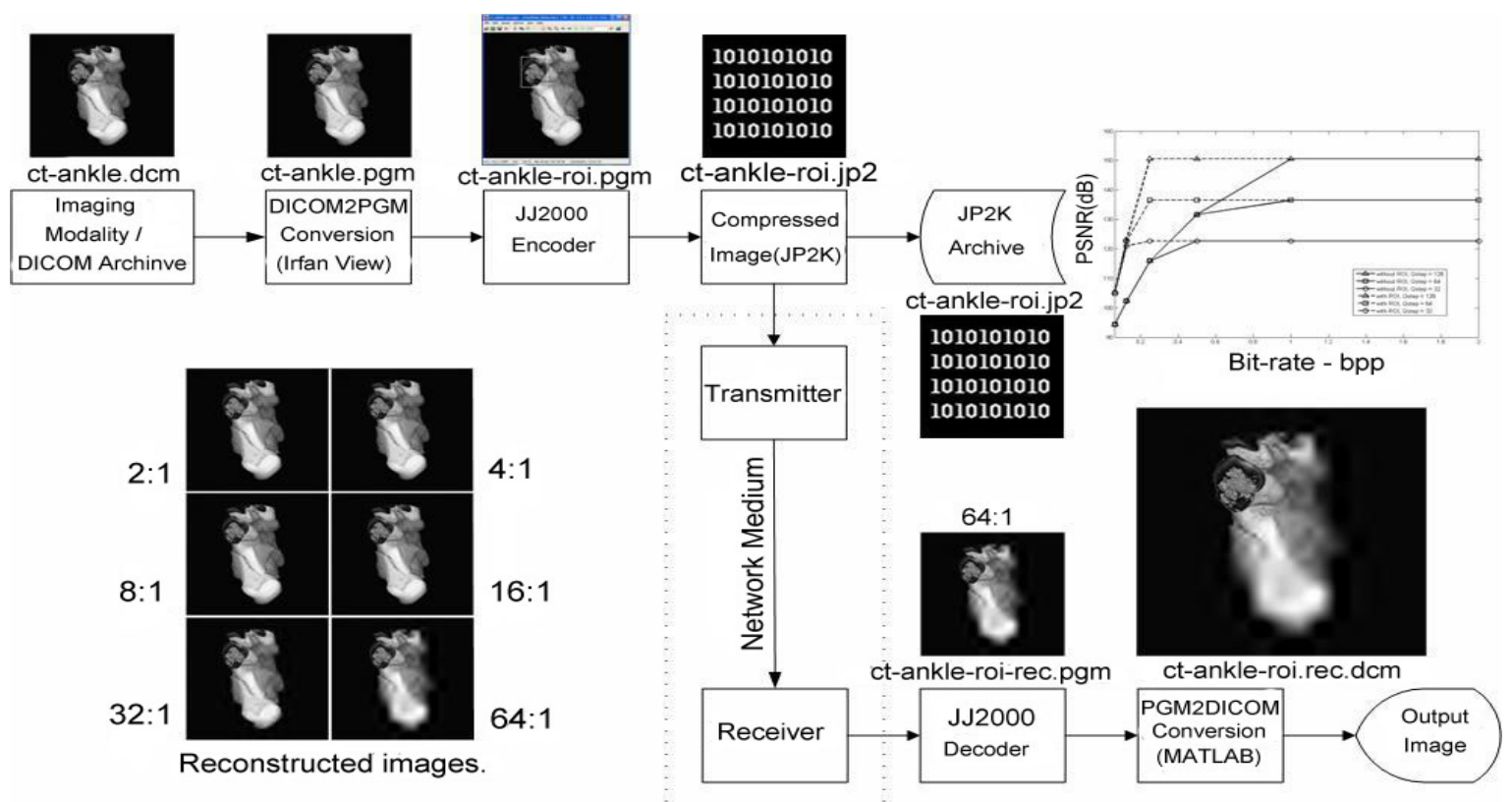

Figure 5. Application of the compression model in Fig. 4 to a CT image.

\subsection{Subjective and Objective Measurements}

The evaluation of the reconstructed images was based upon mixed criteria including: Mean Opinion Score (MOS) [18] for which image quality assessment was carried out by visually comparing the specific ROI of the original and reconstructed images, after the application of the above mentioned compression techniques. The images were presented to six radiologists in a random order. The observers were asked to evaluate the reconstructed images in accordance with their diagnostic value. The ranking was done on an integer scale based on Moving Picture Quality Metric (MPQM) model [19] from 1 to 5, that is, 1 (bad), 2 (poor), 3 (fair), 4 (good) and 5 (excellent). An image is ranked as acceptable if it maintains satisfactory diagnostic value (MOS $\geqslant 4$ ).

When reconstructed images to be encoded contain ROI, Peak Signal-to-Noise Ratio (PSNR) is calculated for the ROI alone and over whole image (for the ROI and the background). For all of the ROI experiments a five level 

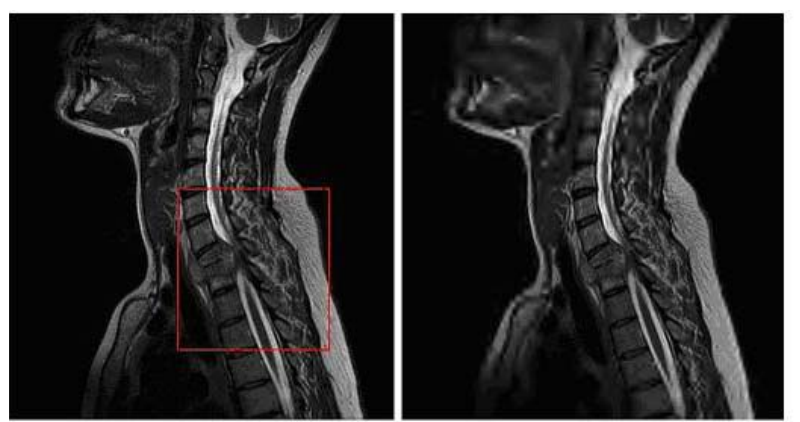

Figure 6. (Left) MRI image with ROI marked in red color and (right) the reconstructed image at the quantization level 1/128 and compression ratio 16:1.

DWT is used and all of the coefficients from the lowest level are included in the ROI. Experiment was completed using JJ2000. The effect of JP2K compression standard on MRI images for lossless compression ratio is presented in Figure 6. The images, at first, are compressed and decompressed at 0.08 bpp up to 4.0 bpp (128:1, 64:1, $32: 1,16: 1,8: 1,4: 1$ and 2:1 compression ratios) at 1/128, $1 / 64$ and $1 / 32$ quantization levels.

\subsection{Discussion}

The MRI image in Figure 6 with ROI (cranial blockage) marked in red color is initially obtained from MRI modality and archived in a DICOM imaging database which is later converted to Portable Gray Map (PGM) format through IrfanView for encoding with JJ2000 software. The image then passed through various stages of the JP2K ROI coding algorithm and finally we get a compressed image ready for storage or transmission. The reconstructed image then followed a similar pattern in reverse when a compressed image is received or accessed from archival. The size of ROI is less than one fourth and typically about $1 / 6$ of the original image.

The superiority of the ROI coding scheme, based on the MAXSHIFT method, over without ROI, can be subjectively and objectively judged at different quantization levels. At 1/128 quantization level achieved a compression ratio of $16: 1$, and subjectively got MOS > 4 and objectively gained up to $20.31 \mathrm{~dB}$, See Figure 7. At 1/64 quantization level with same compression ratio, subjectively got MOS $\geqslant 4$, and objectively gained up to $9.31 \mathrm{~dB}$. At 1/32 quantization level with same compression ratio, subjectively got $\mathrm{MOS}<4$, and objectively show no gain.

Our results show that $16: 1$ compression ratio on $1 / 128$ quantization level using 32x32 code block size, with gain exceeding $18 \mathrm{db}$ appropriate for the MRI images to be reconstructed in lossless settings reducing transmission sixteen times.

\section{CONCLUSIONS}

Our results of reconstructed medical images quality, subjectively and objectively, have shown that the application of JP2K standard based on DWT compression technique with ROI coding using MAXSHIFT scaling method proved diagnostically significant in MRI medical imagery and helpful in identifying the diseases zone. A gain exceeded $18 \mathrm{~dB}$ at 1/128 quantization level achieved with minimum transmission time through various network medium. The results have shown MRI images compression ratio 16:1, acceptable, and may be employed for diagnostically lossless transmission in a teleradiologytelemedicine scenario. In future, we will be preparing our implementation (model) for real-time transmission of diagnostic imagery through automating of image format conversions and simulate on a software/hardware environment. We are also interested in exploration of other radiological areas with rich radiological contents in computed radiography like Mammography.

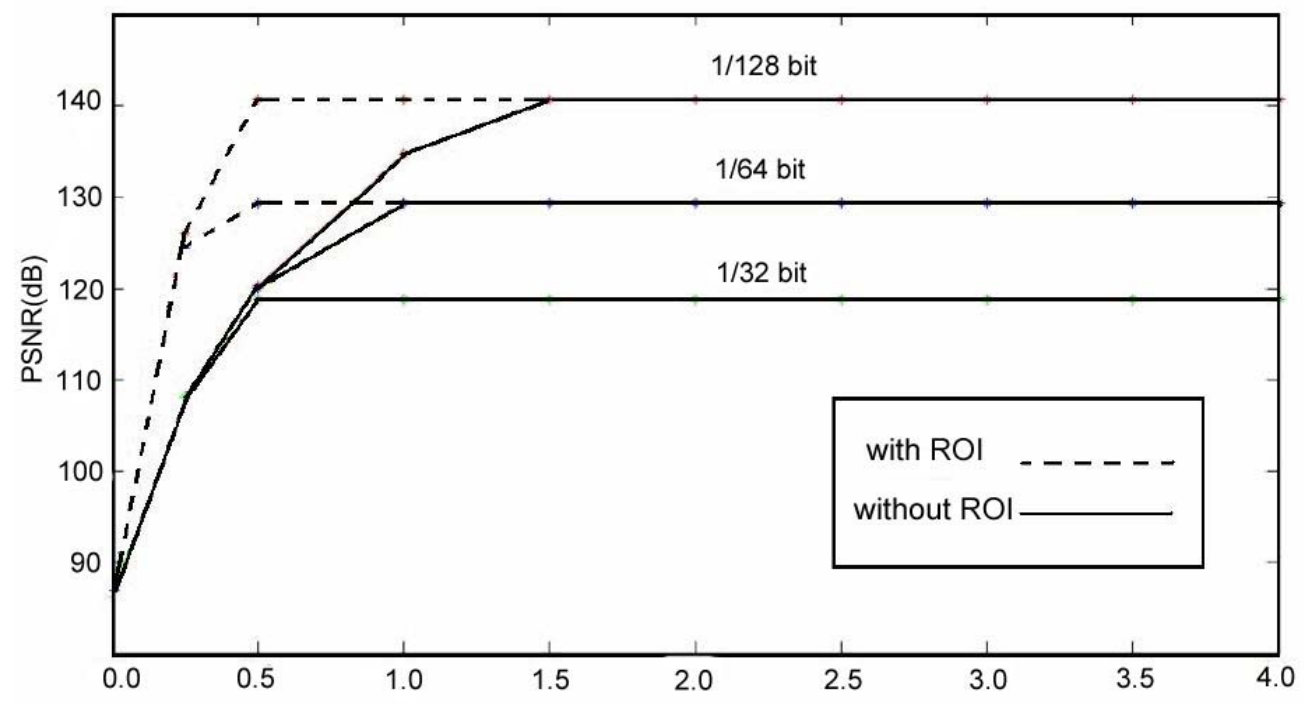

Figure 7. Objective measurement for MRI image in Fig. 6, See subsection 4.3 for details. 


\section{ACKNOWLEDGEMENTS}

We acknowledge radiology departments of Aga Khan University Hospital Karachi, Pakistan, as well as Sindh Institute of Urology and Transplantation, Karachi, Pakistan, for their consistent support without which this work would not be achievable.

\section{REFERENCES}

[1] M. Moore. (1993) Elements of Success in Telemedicine Projects. Report of a research grant from AT\&T Graduate School of Library and Information Science, the University of Texas at Austin.

[2] B.S. Bedi. (2004) Standardization Telemedicine: National Initiative in India. ATA-2004, Tampa.

[3] S. Grimes. (2007) Clinical Engineers: Stewards of Healthcare Technologies. IEEE Engineering in Medicine and Biology Magazine, 23(3), 56-58.

[4] C. Christopoulos, A. Skodras, \& T. Ebrahimi. (2000) The JPEG2000 Still Image Coding System: An Overview. IEEE Transactions on Consumer Electronics, 46, 1103-1127.

[5] M.D. Srinath. (2005) Image Processing: A Clinical Radiologist Perspective. Proceedings of National Conference on Image Processing, Bangalore, India.

[6] P.N. Jayakumar. (2005) Challenges in Medical Image Processing. Proceedings of National Conference on Image Processing, Bangalore, India.

[7] S.T. ChandraShekar \& G.L. Varanasi. (2005) Region of Interest Coding in Medical Images using Diagnostically Significant Bitplanes. Proceedings of Conference on Emerging Aspects of Clinical Data Analysis, Italy.

[8] J. Carrino. (2003) Digital Image Quality: A Clinical Perspective. Quality assurance. The Society for Computer Applications in Radiology, Great Falls, VA, 29-37.

[9] C. Christopoulos, J. Askelf, \& M. Larsson. (2000) Efficient methods for encoding regions of interest in the upcoming JPEG2000 still image coding standard. IEEE Signal Processing Letters, 7(9), 247249.

[10] S. Burak, C. Tomasi, B. Girod, \& C. Beaulieu. (2001) Medical Image Compression based on Region of Interest with Application to Colon CT images. Proceedings of $23^{\text {rd }}$ International Conference of the IEEE Engineering in Medicine and Biology Society, Istanbul, Turkey, 2453-2456.

[11]K. Varma \& A. Bell. (2004) JPEG2000 - Choices and Tradeoffs for Encoders. IEEE Signal Processing Magazine, 21(6), 70-75.

[12]R.S. Kalawsky. (2000) The Validity of Presence as a Reliable Human Performance Metric in Immersive Environments. $3^{\text {rd }}$ International Workshop on Presence, Delft, Netherlands.

[13]M. Boliek. (2000) JPEG 2000 Part I Final Committee Draft Version 1.0 .

[14]D. Leotta \& Y. Kim. (1993) Requirements for picture archiving and communications. IEEE Engineering in Medicine and Biology Magazine, 12(1), 62-69.

[15]I. Skiljan. (2004) Irfanview. Vienna University of Technology.

[16]R. Gonzalez, R. Woods, \& S. Eddins. (2004) Digital image processing using Matlab, Pearson Education, Inc.

[17]D. Santa-Cruz, R. Grosbois, \& T. Ebrahimi. (2003) JJ2000: The JPEG 2000 reference implementation in Java. Proceedings of the First International JPEG 2000 Workshop, Lugano, Switzerland, 4649.

[18]T. Kratochvil \& P. Simicek. Utilization of MATLAB for Picture Quality Evaluation. Institute of Radio Electronics, Brno University of Technology, Brno.

[19] M. Adams \& F. Kossentini. (2000) Reversible integer-to-integer wavelet transforms for image compression: performance evaluation and analysis. IEEE Transactions on Image Processing, 9(6), 10101024. 